\title{
Certain subclass of univalent functions involving fractional q-calculus operator
}

Abdul Rahman S. Juma ${ }^{1}$, Raad AwadHameed ${ }^{2}$ and Mustafa Ibrahim Hameed ${ }^{3}$

${ }^{1}$ University of Anbar, Department of Mathematics, Ramadi-Iraq, dr_juma@hotmail.com

${ }^{2}$ University of Tikrit-Iraq, Department of Mathematics, awad.raad2@gmail.com

${ }^{3}$ University of Tikrit-Iraq, Department of Mathematics, mustafa8095@yahoo.com

\section{Abstract}

The main object of the present paper is to introduce certain subclass of univalent function associated with the concept of differential subordination. We studied some geometric properties likecoefficient inequality and nieghbourhood property, the Hadamard product properties and integraloperator meaninequality.

Key words: Differential subordination, Differential superordination, Univalent function, Convex function, Komatu integral operator, Hadamard product.

\section{AMS2010 Mathematics Subject Classification. Primary30C45 1. Introduction and Definitions}

Let $\mathrm{C}$ be complex plane, letU denote the open unit disc in $\mathrm{C}$,

$$
U=\{z \in C:|z|<1\},
$$

and let Sbe the class of all analytic and univalent functions of the form

$$
f(z)=z+\sum_{k=2}^{\infty} a_{k} z^{k} \cdot(z \in U)
$$

For functions $f$ and $g$ ins such that $g(z)$ defined by

$$
g(z)=z+\sum_{k=2}^{\infty} b_{k} z^{k} . \quad(z \in U)
$$

The Hadamard product(or convolution) of $f$ and $g$ is defined by

$$
(\mathrm{f} * g)(\mathrm{z})=\mathrm{z}+\sum_{k=2}^{\infty} a_{k} b_{k} z^{k}=(g * \mathrm{f})(\mathrm{z}) .
$$

Let $\mathrm{H}(\mathrm{U})$ be the class of holomorphic functions in $U$ and let $H[a, k]$ be the subclass of $H(U)$ of the form

$$
H[a, k]=\left\{f \in H(U): f(z)=a+a_{k} z^{k}+a_{k+1} z^{k+1}+\cdots,(z \in U)\right\},
$$

for $a \in C$ and $k \in N=\{1,2, \ldots$,$\} with H_{0} \equiv H[0,1]$ and $H \equiv H[1,1]$.

Let $\mathrm{K}$ be a subset of $\mathrm{S}$ consisting of function $\mathrm{f}$ with the following form

$$
f(z)=z-\sum_{k=2}^{\infty}\left|a_{k}\right| z^{k},
$$

therefore $f$ are univalent and normalized in the open unit disk $U$.

Now, we let $f(z)$ and $g(z)$ be members of $H(U)$. The function $f(z)$ is said to be subordinate to a function $g(z)$ or $g(z)$ is said to be superordinate tof $(z)$, if and only if there exists a Schwarz function $w(z)$ analytic in $U$, with $w(0)=0$ and $|w(z)|<1,(z \in$ $\mathrm{U})$,

such that

$$
f(z)=g(w(z)),
$$

written as

$$
f \prec \operatorname{gorf}(z) \prec g(z) \quad(z \in U) \text {. }
$$

Furthermore, if the function $g$ is univalent in $U$, then we get the following equivalence $f(z) \prec g(z)$ if and only if $f(0)=g(0)$ and $\mathrm{f}(\mathrm{U}) \subset \mathrm{g}(\mathrm{U})[3],[7]$.

The linear multiplier fractional q-differintegral operator $\mathcal{L}_{\gamma, \tau}^{\alpha, n}$ introduced by [1] defined as follows.

$$
\begin{gathered}
\mathcal{L}_{\gamma, \tau}^{\alpha, 0} f(z)=f(z), \\
\mathcal{L}_{\gamma, \tau}^{\alpha, 1} f(z)=(1-\tau) \mathcal{L}_{\gamma, \tau}^{\alpha} f(z)+\tau z\left(\mathcal{L}_{\gamma, \tau}^{\alpha} f(z)\right)^{\prime}(\tau \geq 0),
\end{gathered}
$$




$$
\begin{gathered}
\mathcal{L}_{\gamma, \tau}^{\alpha, 2} f(z)=\mathcal{L}_{\gamma, \tau}^{\alpha, 1}\left(\mathcal{L}_{\gamma, \tau}^{\alpha, 1} f(z)\right), \\
\mathcal{L}_{\gamma, \tau}^{\alpha, n} f(z)=\mathcal{L}_{\gamma, \tau}^{\alpha, 1} f\left(\mathcal{L}_{\gamma, \tau}^{\alpha, n-1} f(z)\right) . \quad(n \in N)
\end{gathered}
$$

If $f(z)$ is given by $(1.4)$ then by $(1.5)$, we get

$$
\mathcal{L}_{\gamma, \tau}^{\alpha, n} f(z)=z-\sum_{k=2}^{\infty}\left(\frac{\Gamma_{\gamma}(2-\alpha) \Gamma_{\gamma}(k+1)}{\Gamma_{\gamma}(2) \Gamma_{\gamma}(k+1-\alpha)}\left[1-\tau+[k]_{\gamma} \tau\right]\right)^{\alpha}\left|a_{k}\right| z^{k},
$$

where

$$
\mu_{k}^{\alpha}=\left(\frac{\Gamma_{\gamma}(2-\alpha) \Gamma_{\gamma}(k+1)}{\Gamma_{\gamma}(2) \Gamma_{\gamma}(k+1-\alpha)}\left[1-\tau+[k]_{\gamma} \tau\right]\right)^{\alpha} .
$$

By (1.6) and (1.7), then we have

$$
\mathcal{L}_{\gamma, \tau}^{\alpha, n} f(z)=z-\sum_{k=2}^{\infty} \mu_{k}^{\alpha}\left|a_{k}\right| z^{k} \text {. (1.8) }
$$

Note that, if we put $\alpha=0$ the operator $\mathcal{L}_{\gamma, \tau}^{\alpha, n}$ reduces to the operator studied by AL-Oboudi [2] and for $\alpha=0, \tau=1$, we get the operator introduced by S`al`agean [9].

Definition 1.1.A function $f(z) \in K$ and $\theta \geq 0$ the $\theta$ - neighborhood $\mathrm{f}$ is defined as,

$N_{K, \theta}(f)=\left\{g(z)=\mathrm{z}-\sum_{k=2}^{\infty}\left|b_{k}\right| z^{k} \in K ; \sum_{k=2}^{\infty} k|| a_{k}|-| b_{k}|| \leq \theta\right\}$.

In particular, for the function $e(z)=z$, we see that,

$N_{K, \theta}(e)=\left\{g(z)=\mathrm{z}-\sum_{k=2}^{\infty}\left|b_{k}\right| z^{k} \in K ; \sum_{k=2}^{\infty} k\left|b_{k}\right| \leq \theta\right\}$.

The concept of neighborhoods was investigated by Goodman [4]and then generalized by Ruscheweyh [8] , and studied by some authors, A. R. S. Juma and S. R. Kulkarni[5].

Definition 1.2. A function $f(z)$ belonging to $K$ is in the class $K(\alpha, \lambda, n, Y, T, A, B)$, if it satisfies the following

$$
1+\frac{z\left(\mathcal{L}_{\gamma, \tau}^{\alpha+\lambda, n} f(z)\right)^{\prime \prime}}{\left(\mathcal{L}_{\gamma, \tau}^{\alpha, n} f(z)\right)^{\prime}} \prec \frac{1+A z}{1+B z}, \quad(-1 \leq B<A \leq 1)
$$

and is equivalent to the following condition:

$$
\left|\frac{\frac{z\left(\mathcal{L}_{\gamma, \tau}^{\alpha+\lambda, n} f(z)\right)^{\prime \prime}}{\left(\mathcal{L}_{\gamma, \tau}^{\alpha, n} f(z)\right)^{\prime}}}{B \frac{z\left(\mathcal{L}_{\gamma, \tau}^{\alpha+\lambda, n} f(z)\right)^{\prime \prime}}{\left(\mathcal{L}_{\gamma, \tau}^{\alpha, n} f(z)\right)^{\prime}}+(B-A)}\right|<1, \quad(Z \in U) .
$$

Then, we can write

$$
K(\alpha, \lambda, n, Y, T, 1-2 \beta,-1)=K(\alpha, \lambda, n, \gamma, \tau, \beta),
$$

where $K(\alpha, \lambda, n, Y, T, \beta)$ denoted the class of functions in $K$ satisfying the convex inequality:

$$
\Re e\left\{1+\frac{z\left(\mathcal{L}_{\gamma, \tau}^{\alpha+\lambda, n} f(z)\right)^{\prime}}{\left(\mathcal{L}_{\gamma, \tau}^{\alpha, n} f(z)\right)^{\prime}}\right\}>\beta, \quad(0 \leq \beta<1 ; z \in U) .
$$

\section{Main results}

An this section, we derive the coefficient inequality for the $\operatorname{classK}(\alpha, \lambda, n, Y, T, A, B)$.

Theorem2.1. A function $f \in K$ belong to the class $K(\alpha, \lambda, n, \gamma, T, A, B)$ if and only if

$$
\sum_{k=2}^{\infty} k(2 k-1) \mu_{k}^{\alpha}(1-B) \mu_{k}^{\lambda}+(A-B)\left|a_{k}\right| \leq(A-B),
$$

for $\alpha, \lambda, n \in N_{0}, \tau \leq n+1, \gamma \geq 0$ and $-1 \leq B<A \leq 1$. 
Proof.If $f(z) \in K(\alpha, \lambda, n, y, t, A, B)$, then by (1.11), we obtain

$$
1+\frac{z\left(\mathcal{L}_{\gamma, \tau}^{\alpha+\lambda, n} f(z)\right)^{\prime \prime}}{\left(\mathcal{L}_{\gamma, \tau}^{\alpha, n} f(z)\right)^{\prime}} \prec \frac{1+A z}{1+B z},
$$

thus, there exists an analytic function $h(z)$ defined by

$$
h(z)=\frac{z\left(\mathcal{L}_{\gamma, \tau}^{\alpha+\lambda, n} f(z)\right)^{\prime \prime}}{B z\left(\mathcal{L}_{\gamma, \tau}^{\alpha+\lambda, n} f(z)\right)^{\prime \prime}+(B-A)\left(\mathcal{L}_{\gamma, \tau}^{\alpha, n} f(z)\right)^{\prime}} .
$$

Therefore,

$$
\begin{aligned}
& |h(z)|=\left|\frac{z\left(\mathcal{L}_{\gamma, \tau}^{\alpha+\lambda, n} f(z)\right)^{\prime \prime}}{B z\left(\mathcal{L}_{\gamma, \tau}^{\alpha+\lambda, n} f(z)\right)^{\prime \prime}+(B-A)\left(\mathcal{L}_{\gamma, \tau}^{\alpha, n} f(z)\right)}\right| \\
& \left|\frac{-\sum_{k=2}^{\infty} k(k-1) \mu_{k}^{\alpha+\lambda}\left|a_{k}\right| z^{k}}{(B-A) z-\sum_{k=2}^{\infty} k^{2} \mu_{k}^{\alpha}\left(B \mu_{k}^{\lambda}+(B-A)\right)\left|a_{k}\right| z^{k}}\right|<1 .
\end{aligned}
$$

Hence,

$$
\left|\frac{\sum_{k=2}^{\infty} k(k-1) \mu_{k}^{\alpha+\lambda}\left|a_{k}\right| z^{k}}{(A-B) z+\sum_{k=2}^{\infty} k^{2} \mu_{k}^{\alpha}\left(B \mu_{k}^{\lambda}+(B-A)\right)\left|a_{k}\right| z^{k}}\right|<1
$$

Thus, we have

$$
\mathfrak{R} e\left\{\frac{\sum_{k=2}^{\infty} k(k-1) \mu_{k}^{\alpha+\lambda}\left|a_{k}\right| z^{k}}{(A-B) z+\sum_{k=2}^{\infty} k^{2} \mu_{k}^{\alpha}\left(B \mu_{k}^{\lambda}+(B-A)\right)\left|a_{k}\right| z^{k}}\right\}<1 .
$$

Let $|z|=r$ and $0<r<1$, since $\mathrm{h}(\mathrm{z})$ is analytic function for $|z|=1$. Then by (2.3), we obtain

$$
\sum_{k=2}^{\infty} k(k-1) \mu_{k}^{\alpha+\lambda}\left|a_{k}\right| r^{k}<(A-B) r+\sum_{k=2}^{\infty} k^{2} \mu_{k}^{\alpha}\left(B \mu_{k}^{\lambda}+(B-A)\right)\left|a_{k}\right| r^{k}
$$

implies that

$$
\sum_{k=2}^{\infty} k(2 k-1) \mu_{k}^{\alpha}\left((1-B) \mu_{k}^{\lambda}+(A-B)\right)\left|a_{k}\right| r^{k} \leq(A-B) r
$$

the required result follows as $r \rightarrow 1$.

Conversely, for $|z|=r$ and $0<r<1$,we get $r^{k}<r$. That is,

$$
\begin{gathered}
\sum_{k=2}^{\infty} k(2 k-1) \mu_{k}^{\alpha}\left((1-B) \mu_{k}^{\lambda}+(A-B)\right)\left|a_{k}\right| r^{k} \leq \sum_{k=2}^{\infty} k(2 k-1) \mu_{k}^{\alpha}\left((1-B) \mu_{k}^{\lambda}+(A-B)\right)\left|a_{k}\right| r^{k} \\
\leq(A-B) r .
\end{gathered}
$$

By (2.1), we get

$$
\begin{aligned}
&\left|\sum_{k=2}^{\infty} k(k-1) \mu_{k}^{\alpha+\lambda}\right| a_{k}\left|z^{k}\right| \leq \sum_{k=2}^{\infty} k(k-1) \mu_{k}^{\alpha+\lambda}\left|a_{k}\right| r^{k} \\
&<(A-B) r+\sum_{k=2}^{\infty} k^{2} \mu_{k}^{\alpha}\left(B \mu_{k}^{\lambda}+(B-A)\right)\left|a_{k}\right| r^{k}<\left|(A-B) z+\sum_{k=2}^{\infty} k^{2} \mu_{k}^{\alpha}\left(B \mu_{k}^{\lambda}+(B-A)\right)\right| a_{k}\left|z^{k}\right| .
\end{aligned}
$$

Then,we obtain 
$\operatorname{andf}(z) \in K(\alpha, \lambda, n, y, T, A, B)$.

$$
1+\frac{z\left(\mathcal{L}_{\gamma, \tau}^{\alpha+\lambda, n} f(z)\right)^{\prime \prime}}{\left(\mathcal{L}_{\gamma, \tau}^{\alpha, n} f(z)\right)^{\prime}}<\frac{1+A z}{1+B z}, \quad(z \in U)
$$

Theorem2.2. If

$$
\theta=\frac{A-B}{3\left(\frac{\Gamma_{\gamma}(2-\alpha) \Gamma_{\gamma}(k+1)}{\Gamma_{\gamma}(2) \Gamma_{\gamma}(k+1-\alpha)}\left[1-\tau+[k]_{\gamma} \tau\right]\right)^{\alpha}\left[(1-B)\left(\frac{\Gamma_{\gamma}(2-\alpha) \Gamma_{\gamma}(k+1)}{\Gamma_{\gamma}(2) \Gamma_{\gamma}(k+1-\alpha)}\left[1-\tau+[k]_{\gamma} \tau\right]\right)^{\lambda}+(A-B)\right]},
$$

then

$$
\mathrm{K}(\mathrm{\alpha}, \lambda, \mathrm{n}, \mathrm{\gamma}, \mathrm{T}, \mathrm{A}, \mathrm{B}) \subset N_{K, \theta}(e) .
$$

Proof. By from (2.1), andf(z) $\in \mathrm{K}(\alpha, \lambda, n, Y, T, A, B)$, then

$$
3 \mu_{2}^{\alpha}\left((1-B) \mu_{2}^{\lambda}+(A-B)\right) \sum_{k=2}^{\infty} k\left|a_{k}\right| \leq(A-B)
$$

Therefore,

$$
3\left(\frac{\Gamma_{\gamma}(2-\alpha) \Gamma_{\gamma}(k+1)}{\Gamma_{\gamma}(2) \Gamma_{\gamma}(k+1-\alpha)}\left[1-\tau+[k]_{\gamma} \tau\right]\right)^{\alpha}\left[(1-B)\left(\frac{\Gamma_{\gamma}(2-\alpha) \Gamma_{\gamma}(k+1)}{\Gamma_{\gamma}(2) \Gamma_{\gamma}(k+1-\alpha)}\left[1-\tau+[k]_{\gamma} \tau\right]\right)^{\lambda}+(A-B)\right]
$$

$\times \sum_{k=2}^{\infty} k\left|a_{k}\right| \leq(A-B)$,

implies that

$\sum_{k=2}^{\infty} k\left|a_{k}\right| \leq \frac{A-B}{3\left(\frac{\Gamma_{\gamma}(2-\alpha) \Gamma_{\gamma}(k+1)}{\Gamma_{\gamma}(2) \Gamma_{\gamma}(k+1-\alpha)}\left[1-\tau+[k]_{\gamma} \tau\right]\right)^{\alpha}\left[(1-B)\left(\frac{\Gamma_{\gamma}(2-\alpha) \Gamma_{\gamma}(k+1)}{\Gamma_{\gamma}(2) \Gamma_{\gamma}(k+1-\alpha)}\left[1-\tau+[k]_{\gamma} \tau\right]\right)^{\lambda}+(A-B)\right]}=\theta$

From (1.10), we have the following result.

$$
\mathrm{K}(\mathrm{\alpha}, \lambda, \mathrm{n}, \mathrm{Y}, \mathrm{T}, \mathrm{A}, \mathrm{B}) \subset N_{K, \theta}(e) .
$$

Definition2.3. Let $g(z)$ is analytic and defined by $g(z)=z-\sum_{k=2}^{\infty}\left|b_{k}\right| z^{k}$ is said to be a member of the class $\mathrm{K}_{\mathrm{c}}(\alpha, \lambda, n, \mathrm{Y}, \mathrm{T}, \mathrm{A}, \mathrm{B})$ if there exist a function $\mathrm{f}(\mathrm{z}) \in \mathrm{K}(\alpha, \lambda, \mathrm{n}, \mathrm{Y}, \mathrm{T}, \mathrm{A}, \mathrm{B})$ such that

$$
\left|\frac{g(z)}{f(z)}-1\right| \leq 1-c, \quad(0 \leq c<1, \quad z \in U)
$$

Theorem2.4. Iff(z) $\in \mathrm{K}(\alpha, \lambda, n, \mathrm{Y}, \mathrm{T}, \mathrm{A}, \mathrm{B})$ and

$$
\mathrm{C}=1-\frac{3 \theta \mu_{2}^{\alpha}\left((1-B) \mu_{2}^{\lambda}+(A-B)\right)}{6 \mu_{2}^{\alpha}\left((1-B) \mu_{2}^{\lambda}+(A-B)\right)-(A-B)},
$$

then $N_{K, \theta}(f) \subset \mathrm{K}_{c}(\alpha, \lambda, \mathrm{n}, \mathrm{Y}, \mathrm{T}, \mathrm{A}, \mathrm{B})$.

Proof. Let $g(z) \in N_{K, \theta}(f)$. By (1.9), we obtain that

$$
\sum_{k=2}^{\infty} k|| a_{k}|-| b_{k}|| \leq \theta
$$

implies that

$$
\sum_{k=2}^{\infty}|| a_{k}|-| b_{k}|| \leq \frac{\theta}{2}
$$

Since $f(z) \in K(\alpha, \lambda, n, Y, T, A, B)$, by (2.1), we get 


$$
\sum_{k=2}^{\infty}\left|a_{k}\right| \leq \frac{A-B}{6 \mu_{2}^{\alpha}\left((1-B) \mu_{2}^{\lambda}+(A-B)\right)}
$$

where

$$
\begin{aligned}
& \mu_{k}^{\alpha}=\left(\frac{\Gamma_{\gamma}(2-\alpha) \Gamma_{\gamma}(k+1)}{\Gamma_{\gamma}(2) \Gamma_{\gamma}(k+1-\alpha)}\left[1-\tau+[k]_{\gamma} \tau\right]\right)^{\alpha}, \\
& \mu_{k}^{\lambda}=\left(\frac{\Gamma_{\gamma}(2-\alpha) \Gamma_{\gamma}(k+1)}{\Gamma_{\gamma}(2) \Gamma_{\gamma}(k+1-\alpha)}\left[1-\tau+[k]_{\gamma} \tau\right]\right)^{\lambda},
\end{aligned}
$$

thus

$$
\begin{aligned}
& \left|\frac{g(z)}{f(z)}-1\right|=\left|\frac{\sum_{k=2}^{\infty}\left(\left|a_{k}\right|-\left|b_{k}\right|\right) z^{k}}{z-\sum_{k=2}^{\infty}\left|a_{k}\right| z^{k}}\right|<\frac{\sum_{k=2}^{\infty}|| a_{k}|-| b_{k}||}{1-\sum_{k=2}^{\infty}\left|a_{k}\right|} \\
& \leq \theta \frac{3 \mu_{2}^{\alpha}\left((1-B) \mu_{2}^{\lambda}+(A-B)\right)}{6 \mu_{2}^{\alpha}\left((1-B) \mu_{2}^{\lambda}+(A-B)\right)-(A-B)}=1-c .
\end{aligned}
$$

Hence, be Definition 2.3., $g(z) \in \mathrm{K}_{c}(\alpha, \lambda, \mathrm{n}, \mathrm{\gamma}, \mathrm{T}, \mathrm{A}, \mathrm{B})$ for all $\mathrm{c}$ from (2.8).

\section{Hadamard product properties}

In this section we give some properties of the convolution belongs isthe concept.

Theorem3.1. Let the functions $\mathrm{fj}(\mathrm{j}=1,2)$ defined by

$$
f_{j}(z)=z-\sum_{k=2}^{\infty}\left|a_{k, j}\right| z^{k},(j=1,2) .
$$

Then $f_{1} * f_{2} \in \mathrm{K}(\alpha, \lambda, \mathrm{n}, \mathrm{\gamma}, \mathrm{T}, \mathrm{A}, \sigma)$, where

$$
\sigma \leq \frac{A k(2 k-1) \mu_{k}^{\alpha}\left((1-B) \mu_{k}^{\lambda}+(A-B)\right)^{2}-(A-B)^{2}\left(A+\mu_{k}^{\lambda}\right)}{k(2 k-1) \mu_{k}^{\alpha}\left((1-B) \mu_{k}^{\lambda}+(A-B)\right)^{2}-(A-B)^{2}\left(1+\mu_{k}^{\lambda}\right)} .
$$

Proof. First, we find the largest $\sigma$ so that

$$
\sum_{k=2}^{\infty} \frac{k(2 k-1) \mu_{k}^{\alpha}\left((1-\sigma) \mu_{k}^{\lambda}+(A-\sigma)\right)}{A-\sigma}\left|a_{k, 1}\right|\left|a_{k, 2}\right| \leq 1 .
$$

Since $f_{j}(z) \in \mathrm{K}(\alpha, \lambda, \mathrm{n}, \mathrm{\gamma}, \mathrm{T}, \mathrm{A}, \mathrm{B})$, we get

$$
\sum_{k=2}^{\infty} \frac{k(2 k-1) \mu_{k}^{\alpha}\left((1-B) \mu_{k}^{\lambda}+(A-B)\right)}{A-B}\left|a_{k, J}\right| \leq 1 . \quad(j=1,2)
$$

By Cauchy - Schwarz inequality, we have

$$
\sum_{k=2}^{\infty} \frac{k(2 k-1) \mu_{k}^{\alpha}\left((1-B) \mu_{k}^{\lambda}+(A-B)\right)}{A-B} \sqrt{\left|a_{k, 1}\right|\left|a_{k, 2}\right|} \leq 1 .
$$

We show that,

$$
\frac{k(2 k-1) \mu_{k}^{\alpha}\left((1-\sigma) \mu_{k}^{\lambda}+(A-\sigma)\right)}{A-\sigma}\left|a_{k, 1}\right|\left|a_{k, 2}\right| \leq \frac{k(2 k-1) \mu_{k}^{\alpha}\left((1-B) \mu_{k}^{\lambda}+(A-B)\right)}{A-B} \sqrt{\left|a_{k, 1}\right|\left|a_{k, 2}\right| .}
$$

Thus, implies that 


$$
\sqrt{\left|a_{k, 1}\right|\left|a_{k, 2}\right|} \leq \frac{(A-\sigma)\left((1-B) \mu_{k}^{\lambda}+(A-B)\right)}{(A-B)\left((1-\sigma) \mu_{k}^{\lambda}+(A-\sigma)\right)}
$$

By (3.3), we get

$$
\sqrt{\left|a_{k, 1}\right|\left|a_{k, 2}\right|} \leq \frac{A-B}{k(2 k-1) \mu_{k}^{\alpha}\left((1-B) \mu_{k}^{\lambda}+(A-B)\right)}
$$

Hence,

$$
\frac{A-B}{k(2 k-1) \mu_{k}^{\alpha}\left((1-B) \mu_{k}^{\lambda}+(A-B)\right)} \leq \frac{(A-\sigma)\left((1-B) \mu_{k}^{\lambda}+(A-B)\right)}{(A-B)\left((1-\sigma) \mu_{k}^{\lambda}+(A-\sigma)\right)}
$$

this equivalently to

$$
\sigma \leq \frac{A k(2 k-1) \mu_{k}^{\alpha}\left((1-B) \mu_{k}^{\lambda}+(A-B)\right)^{2}-(A-B)^{2}\left(A+\mu_{k}^{\lambda}\right)}{k(2 k-1) \mu_{k}^{\alpha}\left((1-B) \mu_{k}^{\lambda}+(A-B)\right)^{2}-(A-B)^{2}\left(1+\mu_{k}^{\lambda}\right)} .
$$

Theorem3.2. Let the functions $f j(j=1,2)$ defined by $(3.1)$ be in the classK( $\alpha, \lambda, n, \gamma, T, A, B)$. Then the function $p$ defined by

$$
p(z)=z-\sum_{k=2}^{\infty}\left(\left|a_{k, 1}\right|^{2}+\left|a_{k, 2}\right|^{2}\right) z^{k},(3.4)
$$

belong to the class $K(\alpha, \lambda, n, \gamma, T, A, \epsilon)$, where

$$
\epsilon \leq \frac{k(2 k-1) \mu_{k}^{\alpha}\left((1-B) \mu_{k}^{\lambda}+(A-B)\right)^{2}-2 \mu_{k}^{\lambda}(A-B)^{2}-2(A-B)^{2}}{A k(2 k-1) \mu_{k}^{\alpha}\left((1-B) \mu_{k}^{\lambda}+(A-B)\right)^{2}+2 A(A-B)^{2}+2 \mu_{k}^{\lambda}(A-B)^{2}} .
$$

Proof.First, we find the largest $\epsilon$ so that

$$
\sum_{k=2}^{\infty} \frac{k(2 k-1) \mu_{k}^{\alpha}\left((1-\epsilon) \mu_{k}^{\lambda}+(A-\epsilon)\right)}{A-\epsilon}\left(\left|a_{k, 1}\right|^{2}+\left|a_{k, 2}\right|^{2}\right) \leq 1 .
$$

Since $f_{j}(z) \in \mathrm{K}(\boldsymbol{\alpha}, \lambda, \mathrm{n}, \mathrm{\gamma}, \mathrm{T}, \mathrm{A}, \mathrm{B})$, we have

$$
\sum_{k=2}^{\infty}\left(\frac{k(2 k-1) \mu_{k}^{\alpha}\left((1-B) \mu_{k}^{\lambda}+(A-B)\right)}{A-B}\right)^{2}\left|a_{k, 2}\right|^{2} \leq\left(\sum_{k=2}^{\infty} \frac{k(2 k-1) \mu_{k}^{\alpha}\left((1-B) \mu_{k}^{\lambda}+(A-B)\right)}{A-B}\left|a_{k, 1}\right|^{2}\right)^{2} \leq 1
$$

and

$$
\sum_{k=2}^{\infty}\left(\frac{k(2 k-1) \mu_{k}^{\alpha}\left((1-B) \mu_{k}^{\lambda}+(A-B)\right)}{A-B}\right)^{2}\left|a_{k, 2}\right|^{2} \leq\left(\sum_{k=2}^{\infty} \frac{k(2 k-1) \mu_{k}^{\alpha}\left((1-B) \mu_{k}^{\lambda}+(A-B)\right)}{A-B}\left|a_{k, 2}\right|\right)^{2} \leq 1 .
$$

Thus, by (3.5) and (3.6), we give

$$
\sum_{k=2}^{\infty} \frac{1}{2}\left(\frac{k(2 k-1) \mu_{k}^{\alpha}\left((1-B) \mu_{k}^{\lambda}+(A-B)\right)}{A-B}\right)^{2}\left(\left|a_{k, 1}\right|^{2}+\left|a_{k, 2}\right|^{2}\right) \leq 1
$$

But $p(z) \in K(\alpha, \lambda, n, \gamma, T, A, \epsilon)$ if and only if

$$
\sum_{k=2}^{\infty}\left(\frac{k(2 k-1) \mu_{k}^{\alpha}\left((1-\epsilon) \mu_{k}^{\lambda}+(A-\epsilon)\right)}{A-\epsilon}\right)\left(\left|a_{k, 1}\right|^{2}+\left|a_{k, 2}\right|^{2}\right) \leq 1 .
$$

By (3.8), satisfies the following inequality 


$$
\frac{k(2 k-1) \mu_{k}^{\alpha}\left((1-\epsilon) \mu_{k}^{\lambda}+(A-\epsilon)\right)}{A-\epsilon} \leq \frac{k(2 k-1) \mu_{k}^{\alpha}\left((1-B) \mu_{k}^{\lambda}+(A-B)\right)^{2}}{2(A-B)^{2}} .
$$

Hence,

$$
\epsilon \leq \frac{k(2 k-1) \mu_{k}^{\alpha}\left((1-B) \mu_{k}^{\lambda}+(A-B)\right)^{2}-2 \mu_{k}^{\lambda}(A-B)^{2}-2(A-B)^{2}}{A k(2 k-1) \mu_{k}^{\alpha}\left((1-B) \mu_{k}^{\lambda}+(A-B)\right)^{2}+2 A(A-B)^{2}+2 \mu_{k}^{\lambda}(A-B)^{2}} .
$$

\section{Integral Mean Inequalities}

In this section we study the integral meaninequalityby introduce the following definition.

Definition4.1.[10]: The fractional integral of order $s(s>0)$ is defined for a function $f$ by:

$$
D_{z}^{-s} f(z)=\frac{1}{\Gamma(s)} \int_{0}^{z} \frac{f(t)}{(z-t)^{1-s}} d t,
$$

where the function $\mathrm{f}$ is an analytic in a simply connected region of the complex $z$-plane containing the origin, and multiplicity of $(z-t)^{1-s}$ is removed by requiring $\log (z-t)$ to be real, when $(z-t)>0$.

In 1925, Littlewood [6] proved the following subordination theorem:-

Theorem 4.2. (Littlewood [6]): If $f$ and $g$ are analytic in $U$ with $f<g$, then for $\eta>0$

and $z=r e^{i \theta}(0<r<1)$

$$
\int_{0}^{2 \pi}|f(z)|^{n} d \theta \leq \int_{0}^{2 \pi}|g(z)|^{n} d \theta
$$

Theorem 4.3. Let $f(z) \in K(\alpha, \lambda, n, Y, T, A, B)$ and suppose that $f_{k}$ is defined by

$$
f_{k}(z)=z-\frac{A-B}{k(2 k-1) \mu_{k}^{\alpha}\left((1-B) \mu_{k}^{\lambda}+(A-B)\right)} z^{k},(k \geq 2)
$$

and, we let

$$
\sum_{k=2}^{\infty}(i-v)_{\nu+1}\left|a_{i}\right| \leq \frac{(A-B) \Gamma(k+1) \Gamma(s+v+3)}{k(2 k-1) \mu_{k}^{\alpha}\left((1-B) \mu_{k}^{\lambda}+(A-B)\right) \Gamma(k+s+\nu+1) \Gamma(2-v)},
$$

for $0 \leq v \leq i, s>0$, where $(i-v)_{\nu+1}$ defined by

$$
(i-v)_{\nu+1}=(i-v)(i-v+1) \ldots i,
$$

thus, if there exists an analytic function $q$ defined by

$(q(z))^{k-1}=\frac{k(2 k-1) \mu_{k}^{\alpha}\left((1-B) \mu_{k}^{k}+(A-B)\right) \Gamma(k+s+\nu+1)}{(A-B) \Gamma(k+1)} \times \sum_{i=2}^{\infty}(i-\nu)_{\nu+1} H(i)\left|a_{i}\right| z^{i-1}$,

where $i \geq v$ and

$$
H(i)=\frac{\Gamma(i-v)}{\Gamma(i+s+v+1)}, \quad(s>0, i \geq 2)
$$

then, for $z=r e^{i \theta}$ an $d(0<r<1)$

$$
\int_{0}^{2 \pi}\left|D_{z}^{-s-\nu} f(z)\right|^{\eta} d \theta \leq \int_{0}^{2 \pi}\left|D_{z}^{-s-\nu} f_{k}(z)\right|^{\eta} d \theta \cdot(s>0, \eta>0)
$$


Proof. Let $f(z)=z-\sum_{i=2}^{\infty}\left|a_{i}\right| z^{i}$. By Definition 4.1, we have

$$
\begin{gathered}
D_{z}^{-s-\nu} f(z)=\frac{\Gamma(2) z^{s+\nu+1}}{\Gamma(s+\nu+2)}\left(1-\sum_{i=2}^{\infty} \frac{\Gamma(i-1) \Gamma(s+\nu+2)}{\Gamma(2) \Gamma(i+s+\nu+1)}\left|a_{i}\right| z^{i-1}\right) \\
=\frac{\Gamma(2) z^{s+\nu+1}}{\Gamma(s+\nu+2)}\left(1-\sum_{i=2}^{\infty} \frac{\Gamma(s+\nu+2)}{\Gamma(2)}(i-\nu)_{\nu+1} H(i)\left|a_{i}\right| z^{i-1}\right),
\end{gathered}
$$

where

$$
H(i)=\frac{\Gamma(i-v)}{\Gamma(i+s+\nu+1)}, \quad(s>0, i \geq 2)
$$

we get

$$
0<H(i) \leq H(2)=\frac{\Gamma(2-v)}{\Gamma(s+\nu+3)} .
$$

By (4.1) and Definition 4.1., we have

$$
D_{z}^{-s-\nu} f(z)=\frac{\Gamma(2) z^{s+\nu+1}}{\Gamma(s+\nu+2)}\left(1-\frac{(\mathrm{A}-\mathrm{B}) \Gamma(k+1) \Gamma(s+\nu+2)}{k(2 k-1) \mu_{k}^{\alpha}\left((1-B) \mu_{k}^{\lambda}+(A-B)\right) \Gamma(i+s+\nu+1)} z^{k-1}\right),
$$

therefore, we show that

$$
\begin{gathered}
\int_{0}^{2 \pi}\left|1-\sum_{i=2}^{\infty} \frac{\Gamma(s+\nu+2)}{\Gamma(2)}(i-\nu)_{\nu+1} H(i)\right| a_{i}\left|z^{i-1}\right| d \varphi \\
\leq \int_{0}^{2 \pi}\left|1-\frac{(\mathrm{A}-\mathrm{B}) \Gamma(k+1) \Gamma(s+\nu+2)}{k(2 k-1) \mu_{k}^{\alpha}\left((1-B) \mu_{k}^{\lambda}+(A-B)\right) \Gamma(2) \Gamma(i+s+\nu+1)} z^{k-1}\right| d \rho .
\end{gathered}
$$

in virtue of Theorem 4.2, we have

$$
\begin{gathered}
1-\sum_{i=2}^{\infty} \frac{\Gamma(s+v+2)}{\Gamma(2)}(i-v)_{\nu+1} H(i)\left|a_{i}\right| z^{i-1} \\
<1-\frac{(\mathrm{A}-\mathrm{B}) \Gamma(k+1) \Gamma(s+\nu+2)}{k(2 k-1) \mu_{k}^{\alpha}\left((1-B) \mu_{k}^{\lambda}+(A-B)\right) \Gamma(2) \Gamma(i+s+\nu+1)} z^{k-1} .
\end{gathered}
$$

Hence,

$$
\begin{gathered}
1-\sum_{i=2}^{\infty} \frac{\Gamma(s+\nu+2)}{\Gamma(2)}(i-\nu)_{\nu+1} H(i)\left|a_{i}\right| z^{i-1} \\
=1-\frac{(\mathrm{A}-\mathrm{B}) \Gamma(k+1) \Gamma(s+\nu+2)}{k(2 k-1) \mu_{k}^{\alpha}\left((1-B) \mu_{k}^{\lambda}+(A-B)\right) \Gamma(2) \Gamma(i+s+\nu+1)}(q(z))^{k-1},
\end{gathered}
$$

such that

$$
(q(z))^{k-1}=\frac{k(2 k-1) \mu_{k}^{\alpha}\left((1-B) \mu_{k}^{\lambda}+(A-B)\right) \Gamma(k+s+\nu+1)}{(A-B) \Gamma(k+1)} \times \sum_{i=2}^{\infty}(i-\nu)_{\nu+1} H(i)\left|a_{i}\right| z^{i-1}
$$

$q(0)=0$, then we obtain

$$
(q(z))^{k-1} \leq \frac{k(2 k-1) \mu_{k}^{\alpha}\left((1-B) \mu_{k}^{\lambda}+(A-B)\right) \Gamma(k+s+\nu+1)}{(A-B) \Gamma(k+1)} \times \sum_{i=2}^{\infty}(i-\nu)_{\nu+1} H(i)\left|a_{i} \| z\right|^{i-1}
$$




$$
\begin{gathered}
\leq \frac{k(2 k-1) \mu_{k}^{\alpha}\left((1-B) \mu_{k}^{\lambda}+(A-B)\right) \Gamma(k+s+\nu+1)}{(A-B) \Gamma(k+1)} \times H(2)|z| \sum_{i=2}^{\infty}(i-\nu)_{\nu+1}\left|a_{i}\right| \\
=|z| \frac{k(2 k-1) \mu_{k}^{\alpha}\left((1-B) \mu_{k}^{\lambda}+(A-B)\right) \Gamma(k+s+\nu+1)}{(A-B) \Gamma(s+\nu+3) \Gamma(k+1)} \\
\times \Gamma(2-\nu) \sum_{i=2}^{\infty}(i-v)_{\nu+1}\left|a_{i}\right| \leq|z|<1 .
\end{gathered}
$$

If $v=0$, in the Theorem 4.3, we get the following corollary:

Corollary4.4. Let the functions $f(z)$ in the class $\in K(\alpha, \lambda, n, \gamma, T, A, B)$, and suppose that $f_{k}$ is defined by (4.1), and let

$$
\sum_{i=2}^{\infty} i\left|a_{i}\right| \leq \frac{(A-B) \Gamma(k+1) \Gamma(s+3)}{k(2 k-1) \mu_{k}^{\alpha}\left((1-B) \mu_{k}^{\lambda}+(A-B)\right) \Gamma(2) \Gamma(k+s+1)}, \quad k \geq 2
$$

if $q$ defined by

$$
(q(z))^{k-1}=\frac{k(2 k-1) \mu_{k}^{\alpha}\left((1-B) \mu_{k}^{\lambda}+(A-B)\right) \Gamma(k+s+1)}{(A-B) \Gamma(k+1)} \times \sum_{i=2}^{\infty} i H(i)\left|a_{i}\right| z^{i-1},
$$

where

$$
H(i)=\frac{\Gamma(i)}{\Gamma(i+s+1)}, \quad(s>0, i \geq 2)
$$

for $z=r e^{i \theta}$ and $(0<r<1)$

$$
\int_{0}^{2 \pi}\left|D_{z}^{-s} f(z)\right|^{\eta} d \varphi \leq \int_{0}^{2 \pi}\left|D_{z}^{-s} f_{k}(z)\right|^{\eta} d \varphi . \quad(s>0, \eta>0)
$$

\section{References}

[1] S. Abelman, K. A. Selvakumaran, M. M. Rashidi and S. D. Purohit, Subordination conditions for A class of nonbazilevic type defined by using fractional q-Calculus operator, Facta universitatis(NIS) Math. Inform., Vol 32, No 2, (2017), 255-267.

[2] F.M. Al-Oboudi, On univalent functions defined by a generalized Šal aagean operator, Int. J. Math. Math. Sci.,No. 2528,(2004), 1429-1436.

[3] T. Bulboaca, Differential subordinations and superordinations, Recent Results, Casa Cartii de Stiinta, Cluj-Napoca, (2005).

[4] A.W. Goodman, Univalent functions and non-analytic curves, Proc. Amer. Math. Soc., 8(3), (1975), 598-601.

[5] A. R. S. Juma and S. R. Kullcarni, On univalent function with negative coefficients by using generalized Salagean operator,Filomat, 21(2), (2007), 173-184.

[6]L.E. Littlewood, On inequalities in the theory of functions, Proc. London Math. Soc., 23, (1925), 481-519.

[7] S. S. Miller, P. T. Mocanu, Differential subordinations, Theory and applications, Monographs and Textbooks in Pure and Applied Mathematics, Marcel Dekker, Inc., New York, (2000).

[8] S. Ruscheweyh, Neighborhoods of univalent functions, Proc. Amer. Math. Soc., 81, (1981), 521-527.

[9] G.S. S`al agean, Subclasses of univalent functions, in Complex analysis fifth Romanian-Finnish seminar, Part 1 (Bucharest, 1981), 362-372, Lecture Notes in Math., 1013, Springer, Berlin.

[10] H.M. Srivastava and S. Owa (Eds), Current Topics in Analytic Function Theory, World Scientific Publishing Company, Singapore, (1992). 